\title{
Expert reflection in the process of diagnosis of diseases at the extraction of knowledge
}

\author{
Boris Kobrinskii \\ Laboratory Decision clinical support system \\ of Institute of Modern Information Technologies in Medicine of the Federal Research Center \\ "Computer Science and Control" RAS \\ Moscow, Russia \\ bak@isa.ru
}

\begin{abstract}
Reflection of the expert takes among in the process of forming answers to the questions of the cognitive scientist. In medical diagnostics this process including the analysis of disease dynamics. This procedure can be presented in the form of a formula that includes the fuzzy of pathological manifestations, their relevant and the expert certainty factors. This factor characterizes linguistic (verbal) knowledge and visual holistic images in different time periods of the disease.
\end{abstract}

Keywords - formalized representation of the expert's reflection; certainty factors; medical diagnostics; reflection of disease dynamics; fuzzy of disease manifestations; constructs and reflection.

\section{INTRODUCTION}

In the mathematical theory of reflexive structures Lefebvre [1] considers the behavior of a person living in a $+/$ - world, where there are only two poles: positive and negative. A person manifests an inner unconscious intention to a positive or negative choice. The preferable choice is carried out under the influence of the "image of yourself" and the "image of the situation" that a person develops from his self-assessment and evaluation of his concept of himself and the outside world. The American writer Henry David Thoreau wrote about it metaphorically [2]: "No matter how acute my experiences are, I always feel that some part of me treats them critically; it's not even part of me, but an observer who does not share my experiences and only celebrates them".

Unlike the "image of oneself," the model of itself does not have a reality status for the subject [3]. In the logic of argumentation, as the shows [4], all the main results of V.A. Lefebvre, however many interpretations of evaluations and behavior expand due to the differentiation of indefinite and positive action. Sets of arguments in the decision making are divided into disjoint subsets, each of which reflects some aspect of the value system. In the set of arguments, it is possible to select, according to the modality principle, a lot of knowledge.
The phenomenon of reflexion (in relation to medicine) is the reflection (transformation) of the external world of the disease in a particular person (the perception of pathological manifestations) through the inner world of a person, through the psychic sphere of the doctor and the expert doctor or consultant.

\section{REFLECTIVITY OF THE MEDICAL EXPERT}

Reflection manifests itself not only in the attending physician who makes the decision [5], but also with the expert-physician doctor in the process of extracting knowledge from him for creating an intelligent system for supporting the adoption of diagnostic or therapeutic solutions. Let's consider the problem of making diagnostic decisions in the future. Alter ego not only analyzes and evaluates the first hypothesis, but also forms alternative hypotheses, "proposes" proposals for checking the diagnostic significance of information (signs) and possible solutions caused by differential diagnosis with similar diseases.

In transactional categories of description, individuality is the cumulative potential of ego-states as a condition for selfregulation [6]. There is evidence that an innate information processor is "built" into the human psyche, the function of which is to automatically generate images of oneself and others along with their subjective world. The operation of this processor generates a specific spectrum of human responses that are not consciously controlled and proceed extremely fast ( 1 to 2 milliseconds). This kind of reflection, unlike the traditional one, was called fast reflection [7]. In certain cases, it is possible to observe a very fast reaction of the expert to the questions of the cognitive scientist with the presentation of complex diagnostic constructions.

At the same time, the tendency to generate a line of behavior will be called the law of self-reflection [8]. This law, which generates the line of the mental reasoning of the expert, is defined in the application to medicine, on the one hand, by an individual scheme of traditional diagnostic and therapeutic 
processes, on the other hand, by the character and direction of the questions of the cognitive scientist. These questions can partially deform the habitual order of analyzing specific data on the basis of knowledge that has been formed in the process of a generally accepted diagnostic procedure and personal experience (heuristic knowledge). In the presence of a preliminary textual analysis of literary sources, experts take into account subjectivity as available descriptions (severity of symptoms, time of manifestation, etc.), and their assessments from the standpoint of individual knowledge about this pathology. With the group extraction of reflexive knowledge, not only the presentations caused by questions of the cognitive scientist, but also the statements of fellow experts are exposed. In this case, the variables are additionally corrected in the reflexive system of the main expert by the knowledge of other experts about the phenomenon being studied (manifestation of the disease).

\section{IMAGES AND FUZZY IN MEDICAL PRACTICE}

The model of a reflexive solution is not a utility maximization model. It takes into account the polarity (positive and negative quality) outside the context of utility. But it can also take into account the degree of confidence in the decision. The reliability of experts in the expression of signs is manifested in assessing the similarity or difference with the cognitive mental image, in the conviction or doubt in their knowledge about the phenomenon under consideration, which is due not only to the depth of knowledge, but also to the reflexivity of the subject. In particular, this applies to different figurative representations (situations as images based on multi-component features, visual images). It should be borne in mind that the image can be [9]: 1) based on the memory (mental analogy); 2) the result of the simultaneous effect (pseudo-semantic image); 3) associated with the semantic concepts that generate the hypothesis of a diagnosis when a pathognomonic symptom is found that "performs the function of mapping the whole" is akin to a self-reflexive system [10]; 4) the result of the "emergence" of the visual image (pseudo-visual image of the expert).

Any image (semantic script or visual image) almost always differs in fuzzy [11] or underdetermination (NON-factors by A.S. Narinyani [12]), presented in the form of a linguistic scale, taking into account both the temporal characteristics, and his perception is always connected with reflection. In turn, the physician's reflexion and scientific preferences involuntarily lead to increased attention to certain information (activation of certain signs and images) and to an artificial lowering of the role of other data immersed in the depth of consciousness [13]. These images, driven out of consciousness, can subsequently "float up" and again participate in decision-making. If the image seems incomprehensible, if it irritates and does not finish up to something acceptable from the point of view of equilibrium and symmetry, then it is superseded. It exists somewhere in the subconscious until the intuition happen, there is no insight or insight, and then it becomes clear that this image explains the imperfection of the other analyzed image. Such images recessed to a certain time in the subconscious seem to be not trivial (specific) holistic images with orphan (rare) diseases that do not correspond to the usual notions and are not verbalized without loss of integrity.

\section{CONSTRUCTS AND REFLECTION}

According to G. Kelly [14], everything depends on how a person realizes and interprets a particular phenomenon. It does not matter what we are trying to understand, there are always "constructive alternatives" open to our minds. According to Kelly, people are researchers who, in relation to current events and situations, construct hypotheses, and in the case of their refutation - alternatives to these hypotheses. In medicine, they are based on counter-signs or signs-negations. Constructs for their implementation include a professional component and personal perception, including reflection. A construct is a special subjective tool designed by the person himself, allowing him to perceive and understand the surrounding reality, to evaluate and predict events. Constructs can be both verbal (conscious constructs, which can be expressed by words), and verbal (unconscious constructs, which are used if a person does not have words to express it). To measure personal constructs, a methodical principle of "repertory grids" and a repertoire test of personal constructs was developed. It is important to note the lability of repertory grids. This phenomenon can be noted and the expert.

L.Hjelle and D.Ziegler [15] formulated the concept of the individual's personality as an organized system of more or less important personal constructs that a person uses to interpret the world of experiences and to foresee future events, i. dynamics of changes in time.

\section{REFLECTIVE MODEL OF THE EXPERT}

Such a model in the field of medical diagnostics can be based on a scheme that includes a reflexive self-analysis of differential diagnosis. This is a cognitive representation: in the traditional form, a personal "image of the disease" on the basis of precedent or analogues, intuitive notions about the features of the clinical picture and the relationships between the signs, the transformation of manifestations of the disease in time. These representations are characterized by fuzzy manifestations, represented in natural language in linguistic scales with subsequent translation into numerical form $[0,1]$. In the process of extracting knowledge from an expert (or group of experts), the cognitive scientist asks him a series of questions that are aimed at identifying and clarifying knowledge about the disease(s) needed for diagnosis (differential diagnosis). These questions may be pattern of general, clarifying or sounding, and others, including control questions. Integration of numerous sessions of work with an expert allows you to form the field of knowledge of the problem area. The results of the session, including the reflexive component of experts, can be presented in a formalized form.

It is possible to introduce a cortege $(x)$ that reflects the expert's view of a particular disease or a differential diagnostic range of diseases during a session with a cognitive scientist, including a fuzzy variable $(\mathrm{y} 1,2, \ldots, \mathrm{n})$, characterizing a number of manifestations of the disease, presented by the expert in verbal form at the stage of knowledge extraction (for 
fuzzy signs a specially formed linguistic scale is used). This knowledge is formed by the expert in the process of reflexive analysis of the answer to the questions of the cognitive scientist and is accompanied by certainty factor [16] (cf) [-1, +1 ] about each diagnostic feature. Certainty factor can refer to either linguistic or verbalized knowledge in the form of separate signs or symptom-complexes (which can be equated to scenarios) or to holistic (integral) images of a visual type for a particular disease in a certain age period. Depending on the nature of the information analyzed, transitional options are possible from almost absolute certainty, which characterizes an extremely high degree of certainty, to the maximum expressed doubt (an extremely low degree of certainty). In addition, the expert's reflective system evaluates their relevance (r), taking into account the dignity value: especially important, important, relatively significant, little significant, etc. Subsequently this fuzzy linguistic scale is translated by the cognitive scientist into a numerical form in the range from 0.1 to 1$)$.

Assuming that the variables $(\mathrm{x}, \mathrm{y}, \mathrm{cf}, \mathrm{r})$ are defined on the set of real numbers and complemented by the discrete time characteristic $T_{1,2} \ldots, n$, which reflects the changes in the disease in the dynamics, we get a number of knowledge represented by the expert as a function:

where

$$
x=\Phi\left(y, c_{f}, r, T\right)
$$

$\mathrm{x}$ is a cortege reflecting the expert's presentation of a particular disease or a differential diagnostic range of diseases,

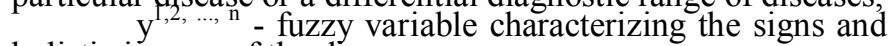
holistic images of the disease,

$c_{f}$ - certainty factor of the expert in the degree of expression of each feature,

$r^{1,2, \ldots, n}$ - the relevance of each sign for the diagnosed disease;

$\mathrm{T}_{1,2, \ldots, \mathrm{n}}$ - time (period) of disease, characterizing the manifestation of signs.

Cortege $(x)$ and the variables $(y 1,2, \ldots, n, c f, r 1,2, \ldots, n)$ depend on $\mathrm{T}$ due to the nature of the manifestation of signs and images in time. When solving a diagnostic task only for a specific point in time or a disease that does not manifest changes in dynamics, the variable $\mathrm{T}$ is not included in the above formula.

\section{THE CONCLUSION}

The reflection takes place at all stages of perception / processing of symptoms, symptom-complexes and images and directs further search for a diagnostic solution along a certain path. However, symptoms in medicine are often characterized by a wide range of manifestations, which corresponds to the concept of fuzzy. The same applies to the image, as well as to the metaphor (for example, Münchausen syndrome in psychiatry) used in medicine, which also differ in fuzzy, i.e. variability of manifestations, and perception of the image or metaphor is always connected with the subject's reflection. This also takes place in the reflection of the logical-andargumentative constructions of the expert, traditionally presented primarily in linguistic form. The analysis of knowledges and datas in dynamics is determined by the age periods of the diseases.
The process of the expert's cogitative activity as a whole, including the expert's reflection, can be implemented in a formalized form and presented on the basis of a formula that includes a fuzzy variable, a confidence factor, relevance of characteristics and a temporal characteristic of changes in the course of diseases.

\section{REFERENCES}

[1] V. Lefebvre, "Choice without criteria of preference", in The structure of human reflexion: The reflexional psychology of Vladimir Lefebvre, $\mathrm{H}$. Wheeler, Eds. New York: Peter Lang Publ, 1990, pp.93-112.

[2] H.D. Thoreau, "Walden or life in the woods", Moscow, Nauka, 1979, 456 pp. (in Rus).

[3] V.A. Lefebvre "The cosmic subject". Moscow: Russian Academy of Sciences, Institute of Psychology Press, 1997, 184 pp. (in Rus).

[4] T.A. Taran, "The problems of conflict modeling and reflexive behavior: the use of the logic of argumentation", VI Intern. Conf. "KnowledgeDialogue-Solution" KDS-97: Collection of scientific papers, vol.1. Yalta, 1997, pp.47-56. (in Rus).

[5] B.A. Kobrinskii, "Reflection and fuzzy representations in medical systems of artificial intelligence", Reflexive control. Intern. sympos. theses. Moscow: Russian Academy of Sciences, Institute of Psychology Press, 1997, pp.85-86. (in Rus).

[6] V.A. Petrovsky, "Individuality and self-regulation: a metamplicative model", in Reflective approach: from methodology to practice, V.E.Lepsky, Eds. Moscow: Kogito-centre, 2009, pp.205-234. (in Rus).

[7] V.A. Lefebvre, "The Fundamental Structures of Human Reflection", J. Soc. Biol. Struct., London, New York, vol.10, iss.2. pp.129-175, April 1987. (references)

[8] V.A Lefebvre, "The Law of Self-Reflexion: A possible unified explanation for three different psychological phenomena", Refl. Processes and Control, vol.1, No.2. pp.91-99, 2002. (references)

[9] B.A. Kobrinskii, "Reflection of image thinking in artificial intelligence systems", VI Intern. Conf. "Knowledge-Dialogue-Solution" KDS-97: Collection of scientific papers, vol.1. - Yalta, 1997, pp.29-36. (in Rus).

[10] V.A Lefebvre, "About self-organizing and self-reflexive systems and their research", Applied ergonomics: Special issue: Reflexive processes. Moscow, no.1, pp.6-9, 1994 (references) (in Rus).

[11] L.A. Zadeh "Fuzzy sets", Inform. and Control., vol.8, iss.3, pp.338-353, June 1965. (references)

[12] A.S. Narinyani "Underdetermination in the system of representation and processing of knowledge", Izv. AN SSSR: Techn. Cyber., Moscow, no.5, pp.3-28, 1986. (references) (in Rus).

[13] D.A. Pospelov "Metaphor, image and symbol in the knowledge of the world", News of artif. Intel., Moscow, no.1, pp. 91-114, 1998. (references) (in Rus).

[14] G. Kelly "Personal Theory (Personal Construct Theory)“, St. Petersburg, Rech, 2000, 249 pp.

[15] L. Hjelle, D. Ziegler "Personal Theory", 3rd intern. ed., St. Petersburg, Piter, 2003, $608 \mathrm{pp}$.

[16] D. Heckerman, E.H. Shortliffe "From certainty factors to belief networks", Artif. Intell. Med., vol.4, iss.1, pp.35-52, February 1992. (references) 